\title{
Comment on Matricardi PM et al.: The first, holistic immunological model of COVID-19: implications for prevention, diagnosis, and public health measures
}

\author{
Antonio Mirijello ${ }^{1}$, Maria D'Errico ${ }^{1}$, Antonella La Marca ${ }^{1}$, Pamela Piscitelli ${ }^{1}$, and \\ Salvatore De Cosmo ${ }^{1}$ \\ ${ }^{1}$ IRCCS Ospedale Casa Sollievo della Sofferenza
}

May 7, 2020

\begin{abstract}
$\mathrm{N} / \mathrm{A}$

Comment on Matricardi PM et al.: The first, holistic immunological model of COVID-19: implications for prevention, diagnosis, and public health measures
\end{abstract}

TO THE EDITOR:

We read with great interest the review article by Matricardi and colleagues [1] depicting mechanisms of disease for COVID-19 and analyzing both viral and host factors influencing its course. We particularly agree with Authors on the pivotal role of innate immunity in the very early phase of disease, being crucial for the subsequent evolution. Most known weapons of innate immune system are represented by natural antibodies, non-specific antimicrobial proteins, interferons, cytokines and cellular elements (i.e. natural killer cells).[1] However, innate immunity could be influenced by other, still underrecognized, factors.

At present, a solid proof of evidence is available on the ability of vitamin D in modulating immune response.[2] Most of data are available from the field of bacterial infections and sepsis, [3] being low vitamin D levels associated with a higher risk of infection and mortality.[4]

In addition, vitamin D could play a role against viruses by maintaining physical barriers (i.e. tight junctions, gap junctions, etc.), enhancing natural immunity (i.e. production of cathelicidin, defensins, etc.) and modulating adaptive immune response (i.e. modulation of TH1/TH2 response and inflammation).[5] On this connection, emerging data support the role of vitamin D supplementation in reducing the risk and severity of influenza.[5] Both influenza and COVID-19 show their maximum spread in winter season and the highest severity in elderly people. Reduced vitamin D levels could represent a possible pathophysiological explanation, among others, in both cases.[5,6] With this regard, it has been hypothesized that variations in vitamin D status across countries and latitudes could, at least in part, explain variations of mortality from COVID-19. [6,7]

However, at present the exact vitamin D status among COVID-19 patients is unknown. [7] Moreover, the role and mechanisms of vitamin D in the treatment of COVID-19 are still unexplored and several interventional trials are ongoing. Should these hypotheses be confirmed, universal vitamin D supplementation would represent a possible and inexpensive strategy to enhance natural immunity against COVID-19.

Antonio Mirijello, MD 
Maria Maddalena D'Errico, MD

Antonella Lamarca, MD

Pamela Piscitelli, MD

Salvatore De Cosmo, MD

Department of Medical Sciences, IRCCS Casa Sollievo della Sofferenza Hospital, San Giovanni Rotondo, Italy

\section{REFERENCES}

1. Matricardi PM, Dal Negro RW, Nisini R. The first, holistic immunological model of COVID-19: implications for prevention, diagnosis, and public health measures. Pediatr Allergy Immunol 2020 May 2. doi: $10.1111 /$ pai.13271.

2. Adams JS, Hewison M. Unexpected actions of vitamin D: new perspectives on the regulation of innate and adaptive immunity. Nat Clin Pract Endocrinol Metab 2008;4:80-90.

3. Mirijello A, Tosoni A, Zaccone V, Impagnatiello M, Passaro G, Vallone CV, et al. MEDS score and vitamin D status are independent predictors of mortality in a cohort of Internal Medicine patients with microbiological identified sepsis. Eur Rev Med Pharmacol Sci 2019;23:4033-4043.

4. Li Y, Ding S. Serum 25-Hydroxyvitamin D and the risk of mortality in adult patients with Sepsis: a meta-analysis. BMC Infect Dis 2020;20:189.

5. Grant WB, Lahore H, McDonnell SL, Baggerly CA, French CB, Aliano JL, et al. Evidence that Vitamin D Supplementation Could Reduce Risk of Influenza and COVID-19 Infections and Deaths. Nutrients 2020;12. pii: E988. doi: 10.3390/nu12040988.

6. Marik PE, Kory P, Varon J. Does vitamin D status impact mortality from SARS-CoV-2 infection? Med Drug Discov 2020 Apr 29:100041. doi: 10.1016/j.medidd.2020.100041.

7. Silberstein M. Vitamin D: A simpler alternative to tocilizumab for trial in COVID-19? Med Hypotheses 2020;140:109767. doi: 10.1016/j.mehy.2020.109767. 